\title{
High pressure insulator-metal transition in $\mathrm{SmB}_{6}$
}

\author{
J.C. Cooley ${ }^{\mathrm{a}, *}$, M.C. Aronson", Z. Fisk ${ }^{\mathrm{b}}$, P.C. Canfield ${ }^{\mathfrak{c}}$ \\ "University' of Michigan, Ann Arbor, MI 48109-1120, USA \\ ${ }^{-}$Los Alamos National Laboratory, Los Alamos. NM 87545, USA \\ 'Ames Laboratory, Iowa State University. Ames, IA 50011, USA
}

\begin{abstract}
We report the temperature and pressure dependence of the electrical resistivity and Hall consiant of single crystal $\mathrm{SmB}_{6}$ for temperatures ranging from $1.2 \mathrm{~K}$ to room temperature, and pressures from 1 bar to $80 \mathrm{kbar}$. Our results indicate that at low pressures $\mathrm{SmB}_{6}$ is an insulator, but undergoes a sudden transition to metallic behavior at a pressure of $50 \mathrm{kbar}$.
\end{abstract}

\section{Introduction}

Recently, there has been extensive theoretical discussion regarding the stability of Kondo insulators under variations in temperature and magnetic field of order the insulating gap [1]. Experimentally, it is a challenge to test these theories by identifying systems in which the insulating gap is both comparable to accessible temperatures and magnetic fields, and also continuosly variable. $\mathrm{SmB}_{6}$ is an ideal candidate for such a study. Previous high pressure resistivity measurements on $\operatorname{Smb}_{6}[2,3]$ found that the application of pressure continuously reduces the observed activation gap, which vanishes in the vicinity of $50 \mathrm{kbar}$. Our experimental strategy is to continuously tune the gap using pressures which approach the critical pressure, and search for signatures in the transport properties suggestive of transitions to new phases. We have simultaneously measured the temperature and pressure dependent Hall voltage of $\mathrm{SmB}_{6}$ allowing the separation of variations in scattering rates and carrier density.

\section{Experimental details}

The samples used in this study are high quality single crystals grown from an aluminum flux. Hall effect and resistivity measurements were carried out using a Bridgman anvil cell, with the applied pressure estimated from force-pressure relations. The Hall voltage and longitudinal magnetoresistance were measured in swept positive and negative fields to correct for contact misalignment voltages.

\section{Experimental results}

The temperature dependences of the electrical resistivity for pressures ranging from 1 bar to $80 \mathrm{kbar}$ are plotted in Fig. 1. Below $48 \mathrm{kbar}$, the electrical resistivity initially decreases with reduced temperature, before sharply increasing and ultimately saturating at the lowest temperatures. The temperature dependence of the resistivity in this pressure range is activated for temperatures

*Corresponding author. 


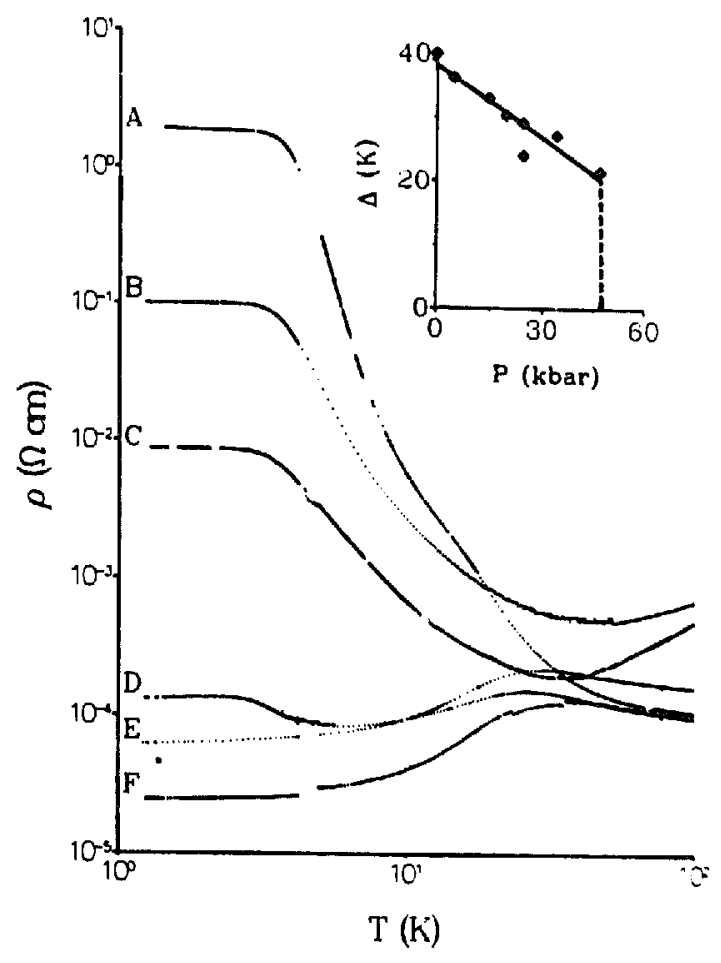

Fig. 1. The temperature dependence of the electrical resistivity of single crystal $\mathrm{SmB}_{6}$ for pressures $A=1$ bar, $B=35 \mathrm{kbar}$, $C=48 \mathrm{kbar}, \mathrm{D}=60 \mathrm{kbar}, \mathrm{E}=70 \mathrm{kbar}$, and $\mathrm{F}=80 \mathrm{kbar}$. Note the qualitative differences in the temperature dependences above and below $48 \mathrm{kbar}$. The inset shows the pressure dependence of the resistivity activation gap. As described in the text, no gap is found above $48 \mathrm{kbar}$.

between 3 and $30 \mathrm{~K}$, if a parallel resistor formulation is used to remove the almost constant saturation resistance observed at the lowest temperatures. The pressure dependence of the activation gap extracted from such fits is plotted in the inset to Fig. 1. The ambient pressure magnitude of the activation gap is $40 \mathrm{~K}$, similar to that found in a variety of previous transport and spectroscopic studies of $\mathrm{SmB}_{6}$ [4-7] but much smaller than that reported in a recent infrared transmission experiment [8].

A dramatic change in the temperature dependence of the resistivity is observed at pressures higher than 48 kbar. Here, the resistivity initially increases with reduced temperature, attaining a maximum at $\sim 25 \mathrm{~K}$. and dropping again at the lowest temperatures. This temperature dependence is reminiscent of metallic mixed valence compounds. There is no appreciable range of temperatures for pressures above $48 \mathrm{kbar}$ where the electrical resistivity exhibits an activated temperature dependence, indicating that there is no gap present in $\mathrm{SmB}_{6}$ above $48 \mathrm{kbar}$. We conclude that while the insulating gap initially decreases linearly with applied pressure, diminished to $22 \mathrm{~K}$ at $48 \mathrm{kbar}$, the gap vanishes suddenly in

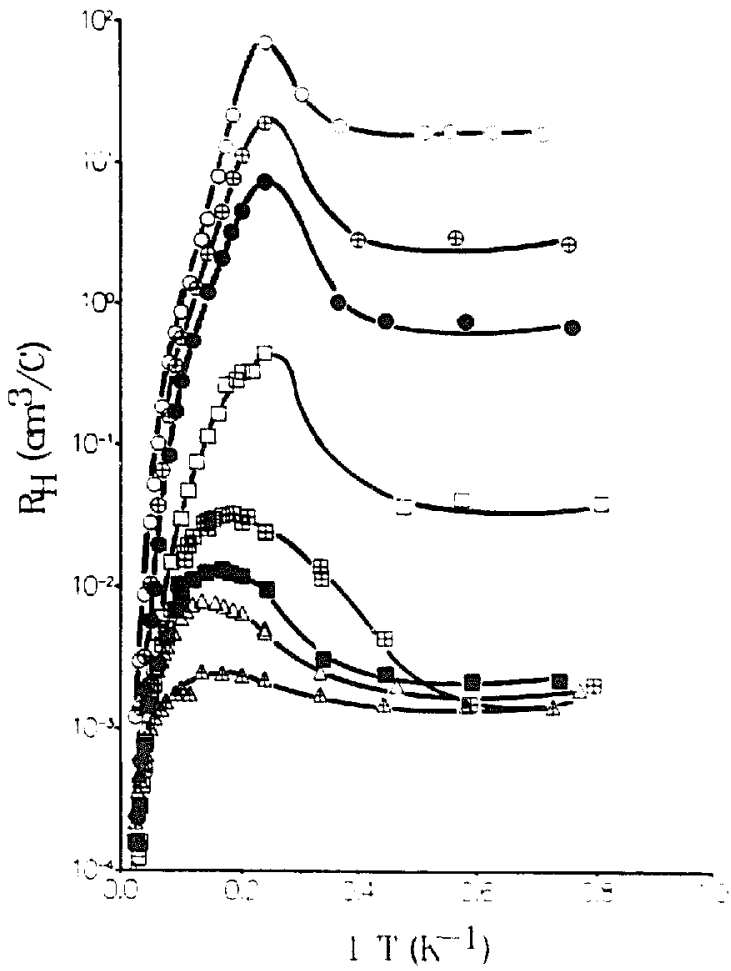

Fig. 2. The temperature dependence of the Hall constant for pressures from 1 bar to $80 \mathrm{kbar}$. $\supset=1 \mathrm{bar}, \oplus=20 \mathrm{kbar}$, - $=25 \mathrm{kbar}, \square=35 \mathrm{kbar}, \square=48 \mathrm{kbar}, \quad \square=60 \mathrm{kbar}$. $\Delta=70 \mathrm{kbar}, \triangle 80 \mathrm{kbar}$. Solid lines are guides for the eye.

a narrow range of pressures near $50 \mathrm{kbar}$. In this respect, our data differ qualitatively from those of previous reports $[2,3]$, which on the basis of more limited data reported a continuous reduction and vanishing of the activation gap near $50 \mathrm{kbar}$.

Further evidence for an abrupt transition from insulating to metallic behavior in the vicinity of $50 \mathrm{kbar}$ is also found in the pressure and temperature dependences of the Hall constant, plotted in Fig. 2. The Hall constant is negative at all temperatures and field independent up to $7 \mathrm{~T}$, initially increasing sharply with reduced temperature, attaining a maximum value near $4 \mathrm{~K}$. before decreasing and becoming constant at the lowest temperatures. The magnitude of the temperature induced changes in the Hall constant is dramatically reduced with the application of pressure. Interestingly, there is no appreciable range of temperatures or pressures on which the Hall constant exhibits activated behavior with the same gap as the resistivity, suggesting that the observed temperature dependence not only depends on the semiconducting background and impurity bands but possibly also reflects the presence of anomalous skew scattering. 


\section{Discussion}

In view of the potentially complex nature of the temperature dependent Hall constant, we focus here on its low temperature properties. We can gain insight into the nature of the low temperature electronic structure by comparing the low temperature, constant values of the residual resistivity $\rho_{0}$ and the carrier population $n_{0}$ derived from the inverse Hall constant. At ambient pressure these quantities are $1.9 \Omega \mathrm{cm}$ and $3.6 \times 10^{17} \mathrm{~cm}^{-3}$, respectively. It is important to note that the maximum resistivity resulting from a single unitarity scatterer per unit cell in $\mathrm{SmR}_{6}$ is only $540 \mu \Omega \mathrm{cm}$ [5] more than three orders of magnitude smaller than the observed ambient pressure $\rho_{0}$. The simultaneous observations of enormous $\rho_{0}$ and small $n_{0}$ at low pressures can be reconciled by postulating that the low temperature transport is dominated by an extended impurity band. With increased pressure, the number of carriers in the impurity band grows and the bandwidth increases, decreasing the associated residual resistivity. Above $50 \mathrm{kbar}$, the carrier number becomes essentially pressure independent, signalling that the impurity band has been absorbed into the conduction band, presumably as a result of the sudden gap closure revealed by the resistivity measurements.

In summary, the pressure dependence of the electrical resistivity and the Hall constant reported here suggest that $\mathrm{SmB}_{6}$ is an insulator at low pressure, but that a sudden transition to metallic behavior similar to that found in metallic Kondo lattice materials is observed when the pressure is increased past $50 \mathrm{kbar}$.

\section{Acknowledgements}

The authors acknowledge useful discussions with $P$.

Schlottmann, J. Rasul, and J.W. Allen. Work at Los
Alamos National Laboratory was performed under the auspices of the US Department of Energy. Ames Laboratory is operated for the US Department of Energy by lowa State University under contract number W-7405Eng-82. Part of this work was supported by the Director for Energy Research, Office of Basic Science.

\section{References}

[1] S. Doniach and P. Fazekas, Phil. Mag. B 65 (1992) 1171; V. Dorin and P. Schlottmann, Phys. Rev. B 46 (1992) 10800; P. Fazekas and E. Muller-Hartmann, Z. Phys. B 85 (1991) 285; A.J. Millis, in: Physical Phenomena at High Magnetic Fields, eds. E. Manousakis, P. Schlottmann, P. Kumar, K. Bedell and F.IM. Mueller (Addison-Wesley, Redwood City, CA, 1992) p. 146.

[2] J. Beille, M.B. Maple, J. Wittig, Z. Fisk and L.E. Delong, Phys. Rev. B 28 (1983) 7397.

[3] I.V. Berman, N.B. Brandt, V.V. Moshchalkov, S.N. Pashkevich, V.I. Sidorov, E.S. Konovalova and Yu. B. Paderno, Pis'ma Zh. Eksp. Teor. Fiz. 38 (1983) 393.

[4] J.C. Nickerson, R.M. White, K.N. Lee, R. Bachmann, T.H. Geballe and G.W. Hull, Phys. Rev, B 3 (1971) 2030.

[5] J.W. Allen, B. Batlogg and P. Wachter, Phys, Rev. B 20 (1979) 4807.

[6] I. Frankowski and P. Wachter, Solid State Commun. 41 (1982) 577.

[7] G. Guntherodt, W.A. Thompson, F. Holtzberg and Z. Fisk, in: Valence Instabilities, eds. P.Wachter and H. Boppart (North-Holland, Amsterdam, 1982) p. 313.

[8] T. Nanba, H. Ohta, M. Motokawa, S. Kimura, S. Kunii and T. Kasuya, preprint (1992). 